\title{
From Molecules to Behavior and the Clinic: Integration in Chronobiology
}

\author{
William Bechtel \\ Department of Philosophy, Center for Chronobiology, and \\ interdisciplinary programs in Cognitive Science and Science Studies \\ University of California, San Diego
}

\begin{abstract}
Chronobiology, especially the study of circadian rhythms, provides a model scientific field in which philosophers can study how investigators from a variety of disciplines working at different levels of organization are each contributing to a multi-level account of the responsible mechanism. I focus on how the framework of mechanistic explanation integrates research designed to decompose the mechanism with efforts directed at recomposition that relies especially on computation models. I also examine how recently the integration has extended beyond basic research to the processes through which the disruption of circadian rhythms contributes to disease, including various forms of cancer. Understanding these linkages has been facilitated by discoveries about how circadian mechanisms interact with mechanisms involved in other physiological processes, including the cell cycle and the immune system.
\end{abstract}

\section{Introduction}

Chronobiology provides a model scientific field ${ }^{1}$ for examining processes of integration in biology. Chronobiology is a relatively small interdisciplinary field that investigates endogenously generated periodic phenomena-biological rhythms. The most thoroughly studied and widely known biological rhythms are circadian rhythms: endogenously generated oscillations manifest in physiological and behavioral processes with periods of approximately 24 hours that are entrainable to the environmental day-night cycle and are temperature compensated. Although I will not consider them here, chronobiology also investigates ultradian rhythms that have periods much shorter than a day (e.g., approximately 90 minute oscillations in metabolism and sleep) as well as infradian rhythms whose periods are longer than a day (e.g., annual cycles of fertility). Perhaps the best known physiological process regulated by circadian rhythms is the sleep-awake cycle, but in fact a wide variety of other physiological processes, including heart rate, blood pressure, body temperature, cell division, metabolism of glucose, lipids, and drugs, hormone regulation, and bone formation, as well as many mental processes, including attention and speed of reactions, exhibit circadian oscillations.

In this paper I will focus on integration within the interdisciplinary field of chronobiology that began historically by investigating rhythms exhibited in behavior and how they could be entrained to the external environment, proceeded by localizing the source of these rhythms in the brain and investigating the oscillatory mechanism underlying these

${ }^{1}$ I use the notion of model field in the same sense in which biologists refer to model organisms and model systems. 
rhythms at the molecular level, and more recently has recognized the need to computationally model both the intracellular mechanisms and how cells synchronize their oscillations and has identified linkages to processes of importance in clinical medicine. Chronobiology researchers are drawn from a wide range of disciplines, including genetics, biochemistry, cell and molecular biology, behavioral biology, psychology, psychiatry, and cancer research. Investigators in this interdisciplinary field collaborate with each other and draw upon research techniques from each of these disciplines. They are highly informed about contributions from the diverse disciplines. A particular noteworthy feature of research in chronobiology is that it employs a wide range of model organismscyanobacteria, fungi (Neurospora crassa), plants (Arabidopsis), and animals (fruit flies and mice). Researchers that specialize on particular model organisms nonetheless keep current on research on the others. This integration is in part enabled by shared journals (especially the Journal of Biological Rhythms) and biennial meetings of the Society for Biological Rhythm Research.

I will argue that integration of such diverse disciplines, techniques, and model organisms is facilitated by the common acceptance of the circadian clock metaphor and the assumption that there is a conserved mechanism responsible for circadian phenomena. Different avenues of research investigate different aspects of the mechanism, some focusing on better articulating the phenomenon for which the mechanism is responsible, others decomposing and then recomposing the mechanism to understand how component parts and operations at different levels of organization work together to generate the phenomena, and yet others addressing how both the phenomenon and the responsible mechanisms interact with other mechanisms that figure in health and disease.

One part of the explanation for the high degree of integration is that chronobiology is a relatively small field of research with a reasonably short history such that splintering of specializations has not occurred (Gerson, this issue). From a founding event in 1960s, the field has maintained a high degree of interaction between the various contributing modes of inquiry. I will briefly explore the early history in section 2 where I will also introduce the important role of the framework of mechanistic explanation that is embodied in the clock metaphor that has pervaded the field. Mechanistic research is often primarily associated with downward or reductionistic research that attempts to take mechanisms apart to identify their parts and operations, and as I will discuss in section 3, this has been one of the major triumphs of chronobiology. To understand a mechanism, however, one must not only identify its parts and operations but also discover how these are organized and integrated to generate the phenomenon. This activity of recomposition I will characterize in section 4 as upwards so as to mark the contrast with the downward direction of decomposition. Just as decomposition can be iterated by decomposing the parts of the initial decomposition, recomposition can be iterated by showing how a mechanism is also a part of yet another mechanism.

In section 5 I develop a common and important facet of mechanistic research that has not yet received extensive discussion - its role in both understanding and treating disease conditions. This includes not just the immediate consequences of disrupted rhythms, such as altered sleep patterns, but diseases that are primarily manifest in physiological functions 
attributed to other mechanisms. Although research on different phenomena frequently begins by assuming the responsible mechanisms are autonomous from each other, subsequent research often reveals how intertwined they are. The recognition of illnesses in individuals suffering chronic jet lag is revealing a large number of interconnections between the circadian clock mechanism and processes involved in cell division, immune responses, and metabolism. As research has identified the relevance of the circadian rhythms to a wide range of diseases, it has motivated further integration efforts directed at understanding how the circadian clock mechanism is linked to other physiological mechanisms.

In Bechtel (1986) I distinguished instances of integration in science that resulted in new disciplines (biochemistry) from those that yielded interdisciplinary research clusters (cognitive science). Although some researchers in cognitive science have tried to advance it as a separate discipline, for the most part researchers continue to identify themselves with one of the contributing disciplines (computer science, cognitive psychology, etc.). In a symposium celebrating the thirtieth anniversary of the Cognitive Science Society the individual talks focused on contributions from the contributing disciplines and the likely future contributions of those disciplines to cognitive science. In this regard, chronobiology is more like cognitive science than biochemistry or cell biology in that it remains an interdisciplinary field. ${ }^{2}$ As Brigandt (2010) shows, these instances of integration that do not involve new disciplinary structures result from active collaboration between researchers in different disciplines pursuing a common problem or epistemic goal. The interdisciplinary field of chronobiology is not limited to pursuit of a single problem or goal but includes inquiry into various phenomena involving biological rhythms; yet it is the

2 The contrast between new disciplines and enduring interdisciplinary fields is not sharp, but is reflected by differences on several dimensions, many of them sociological. Disciplines are typically associated with academic departments that grant degrees, especially doctorate degrees, which perpetuate the population of investigators in the discipline. Academic institutions are sometimes rather coarse-grained in their relation to disciplines: they may have departments of biology rather than departments of cell and molecular biology, genetics, neuroscience, evolution and ecology, etc., but even then their curriculum is often divided into these more finely delineated disciplines. There are no departments of chronobiology; rather, if there is an institutional structure it will have the form of an interdisciplinary center, such as the Center for Chronobiology at UCSD, which serves to foster interaction between researchers situated in a number of different departments. A second dimension involves the type of relation of researchers to professional societies. In North America a given researcher would likely view annual meetings of the American Society for Cell Biology or the Society for Neuroscience as their primary professional meeting. They may participate in more specialized fields and often these will cross disciplinary lines by drawing researchers from multiple disciplines. The Society for Research on Biological Rhythms draws researchers from across a range of disciplines, not just within biology but also from mathematics, engineering, psychology, psychiatry, and other medical specializations. 
interacting research objectives, not the institutional framework of a discipline, that is responsible for the integration.

\section{Chronobiology and the Clock Mechanism Metaphor}

Investigators over many centuries identified biological activities that oscillate with a 24hour period. Androsthenes, a ship captain serving under Alexander the Great, observed that plants such as the tamarind tree open and close their leaves on a daily cycle. Jean Jacques d'Ortous De Mairan (1729) wondered whether such behavior in plants was a mere response to sunlight or originated within the plant and so took the additional step of maintaining Mimosa plants in a dark cupboard at relatively constant temperatures. He found that they still opened and closed their leaves on a daily cycle. Numerous researchers in the $19^{\text {th }}$ century, including Darwin, extended these observations and in the early $20^{\text {th }}$ century animal researchers identified a variety of behaviors that exhibit daily oscillations (e.g., body temperature and sleep in humans, eclosion from pupae in fruit flies, and food seeking behavior in bees). The question Mairan had advanced, whether these oscillations were endogenously controlled or governed by environmental cues, was the focus of many of these investigations. (See Daan, 2010, for a detailed historical review.)

Two of the leading contributors to this research, Pittendrigh and Aschoff, met in the late 1950s and organized an international Symposium on Biological Clocks that met from 5-14 June 1960 at Cold Spring Harbor in the U.S. 150 researchers participated, including 31 from outside the U.S. The abundance of evidence presented at the symposium effectively settled that circadian behaviors are endogenously generated. One of the most compelling forms of evidence was that when light and other cues are removed (producing a condition known as free-running), the oscillatory period typically varies somewhat from 24 hours. This had led Halberg et al. (1959) to coin the term circadian (circa=about + dies=day) for these rhythms. The recognition that endogenous rhythms vary slightly from 24 hours focused attention on the question of how various cues such as light (known as Zeitgebers) entrained the oscillations to the 24-hour light-dark cycle found on Earth. There were no resources at the time for determining how entrainment was accomplished, but investigators were able to delineate the phenomenon of entrainment by, for example, investigating how much light pulses at different times of day either advanced or delayed the phase of circadian oscillations.

Once it was established that circadian oscillations were endogenously controlled, the quest to understand the responsible mechanism became focal. In what became a classic text, Bünning (1958) referred to a physiological clock. ${ }^{3}$ The 1960 symposium that employed the

3 The idea of a clock mechanism has earlier roots. The French pharmacist Julien-Jospef Virey (1814) introduced the term horloge vivante or living clock as a result of his observations of a daily cycle in human mortality. The term physiological clock was employed by Maynard Johnson (1939) in the conclusion of a report on white-footed mice kept in constant light in which he established that the periodicity of the behavior depended on the intensity of the light: "the animal has an exceptionally substantial and durable self- 
term biological clock in its title, and in his opening address Bünning (1960) characterized it as "the timing mechanism by which organisms mark the passage of the hours of the day." As I will discuss in the next section, actual investigation of the clock mechanism would require introducing new techniques beyond the modes of behavioral investigation represented at the symposium, but Bünning effectively set the primary agenda for the field-to identify the clock mechanism and explain how it operates.

In searching for a clock mechanism, circadian researchers were following a well-trodden path in biology-explaining a given phenomenon by identifying and describing the mechanism responsible for it. Although many philosophers of science in the $20^{\text {th }}$ adopted classical physics as their model and focused on laws as the vehicle of explanation, by the end of the century philosophers addressing explanation in biology began to follow the lead of biologists and analyzed how accounts of mechanisms provide explanations. While terminology differs, the central idea is that a mechanism produces a phenomenon of interest as a result of the organized and coordinated operations performed by its parts (Bechtel \& Richardson, 1993/2010; Bechtel \& Abrahamsen, 2005; Machamer et al., 2000).

Philosophers developing accounts of mechanistic explanation have considered not just what a mechanism is, but how accounts of mechanisms are developed, evaluated, and revised over time (Bechtel, 2006; Craver, 2007; Darden, 2006). Much of the emphasis has been on the process of decomposing mechanisms, which is highly fitting since this has been the forte of mechanistic biology as it has developed over the past two hundred years. ${ }^{5}$ Biologists have developed tools for manipulating (lesioning, stimulating, etc.) putative parts of mechanisms and developed inference strategies to determine from the effects of

winding and self-regulating physiological clock, the mechanism of which remains to be worked out."

${ }^{4}$ Bünning commented: "The hardest question in our Symposium will undoubtedly be that of the clockwork itself" (p. 4). He went on to invoke a further feature of circadian rhythms - they are maintained with nearly the same period irrespective of temperatureas a clue to the nature of the mechanism. Since chemical reactions generally speed up significantly as temperature raises, he inferred "This cannot be a case of a simple fluctuation of biochemical reactions in the sense of enzyme controlled processes. The slight temperature dependence of the period length suggests that the clock operates by a physical, rather than a chemical mechanism" (p. 4). Bünning goes on to hypothesize an alteration in the state of macromolecules involving "a succession of tension and relaxation processes" (p. 6). Subsequent research, however, favored a chemical mechanism, and although their have been many attempts to explain how it is temperature compensated, this remains a topic of active investigation.

${ }^{5}$ Bechtel and Richardson (1993/2010) offered decomposition of mechanisms into components as an alternative to the accounts of theory reduction then prominent in philosophy of science that emphasized deduction of theories from lower-level theories (for discussion, see Bechtel \& Hamilton, 2007). More recent accounts of mechanism have emphasized how it provides for the integration of information about processes at multiple levels of organization (Craver, 2005; Bechtel \& Abrahamsen, 2009). For a detailed review of these issues, see Brigandt and Love (2012). 
the manipulations on the behavior the whole what operations the manipulated parts performed. Additionally, they have developed tools for tracing activity in mechanisms by, for example, presenting an input to the mechanism and recording how various parts respond. Although biologists have long been concerned with how the parts operate together in a whole mechanism, the tools for understanding more than relatively simple forms of organization (sequential performance of linearly characterized operations) have been limited. Techniques for mathematically representing and simulating mechanisms are increasingly being adopted by biologists, enabling them to characterize the often complex dynamic behavior of mechanisms by generating what Bechtel and Abrahamsen (2010, 2011) characterize as dynamic mechanistic explanations (for discussion of the explanatory role of computational models, see Brigandt, this issue, in press). Mathematical representations and simulations figure centrally in attempts to recompose mechanisms. Endeavors to recompose often require extending beyond the boundaries of the mechanism. An implicit assumption of mechanistic researchers is that the mechanism they are investigating is effectively segregated from and working largely in isolation from others (in doing so they implicitly adopt what Simon, 1962, characterized as the heuristic of assuming natural systems are nearly decomposable; for illuminating discussion, see Wimsatt, 1974; Wimsatt, 2007). But often as investigations progress researchers discover that the mechanisms they investigate affect and are affected by other mechanisms amongst which they are situated.

As I will develop in subsequent sections, it is the three features of mechanistic research (decomposition, recomposition, and situating) that have rendered it an effective vehicle for integration in the field of chronobiology. The necessary first step in investigating mechanisms is decomposing them into component parts and operations, for without at least an initial identification of parts and operations, there is nothing to recompose or situate. Thus, I turn first to the efforts to decompose the circadian clock.

\section{Localizing and Decomposing the Circadian Clock Mechanism}

The hypothesis that there is a clock mechanism raised the question of where it was located. As Daan (2010) notes, many circadian researchers were initially skeptical of the idea of a localized clock, assuming that the whole body served as the oscillator. However, some animal researchers assumed that the clock would be located in a specific part of the brain and set out to find it experimentally. Richter (1967) showed that lesions to the hypothalamus in mammals could eliminate circadian behavior and was trying to narrow the responsible region when, in 1972, two other research groups identified the suprachiasmatic nucleus (SCN), a bilateral hypothalamic nucleus with approximately 20,000 neurons on each side in the mouse and located just above the optic chiasm, as the locus of the mammalian clock. Focusing on how light would be able to entrain the clock, Moore and Lenn (1972) identified the SCN as a target of projections from the retina whereas Stephan and Zucker (1972) and Moore and Eichler (1972) showed that lesions to it rendered animals arrhythmic. This localization of the clock received further support when Inouye and Kawamura (1979) demonstrated, using multi-electrode recording, that isolated SCN tissue remained rhythmic while the rest of the brain and animal did not and Ralph et al. (1990) succeeded in transplanting the SCN from a mutant hamster exhibiting a 
shortened rhythm into the ventricles of SCN-lesioned hosts and produced shortened rhythms in the recipient.

Localizing a mechanism does not yet explain the phenomenon. Explanation requires showing how the localized mechanism is capable of generating the phenomenon. This necessitates decomposing the mechanism itself, which often requires researchers with different experimental tools. In the circadian case the first major insight was provided not by direct attempts to decompose the SCN, but by genetic research that aimed at identifying responsible genes. Benzer developed a research program that exposed fruit flies to mutagenic agents and then screened them for aberrant traits that he associated with a gene at the locus of mutation. As a graduate student with Benzer, Konopka applied this approach to circadian rhythms in fruit flies, creating mutants that were either arrhythmic or exhibited shortened (20 hour) or lengthened (28 hour) rhythms (Konopka \& Benzer, 1971). He traced all these effects to a mutation at a common location on the $X$ chromosome and named the responsible gene period (per). His success both inspired the search for circadian genes in other species ${ }^{6}$ and attempts to use the mutants to decipher the mechanism.

Although a number of investigators tried to infer the clock mechanism directly from these genetic clues (Edmunds, 1988), the crucial insight required the introduction of cloning techniques that made it possible to measure the concentrations of the mRNA transcript of per and the protein synthesized from it. Using this approach, Hardin, Hall, and Rosbash (1990) demonstrated daily oscillations in both per mRNA and the protein PER, with the protein reaching maximal concentration several hours after the mRNA. From this clue they proposed a transcriptional-translational feedback loop mechanism whereby, once PER was synthesized, it suppressed its own synthesis (Figure 1, left). Only when it was degraded, could more PER be synthesized. They hypothesized that, with appropriate delays for the various stages, this process could generate 24 -hour oscillations.

${ }^{6}$ Konopka himself had identified other loci in fruit flies. Feldman and Hoyle (1973) generated mutations that altered clock behavior in Neurospora and Ralph and Menaker (1988) produced the first mammalian circadian mutant in hamsters (which then figured in their transplant study noted above). 


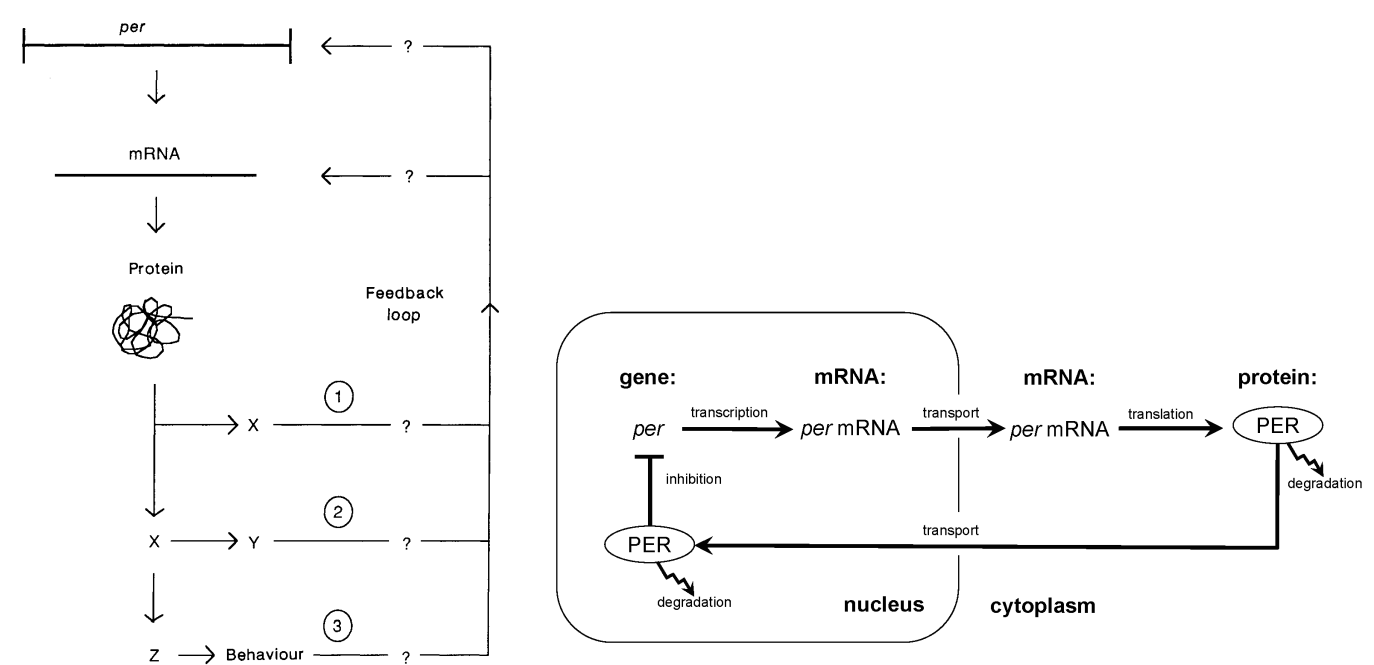

Figure 1. Shown on the left is the mechanism proposed by Hardin et al. (1990) showing various options as to the agent and target of inhibitory feedback. On the right is basic conception of the basic transcription-translation feedback mechanism that soon was generally accepted.

As the question marks in their figure make clear, they contemplated several possibilities for the actual operation of the mechanism: they proposed three options for what actually figured in the negative feedback-PER itself, a protein produced from it, or even behavior produced by the organism - and two options for the target of feedback-transcription or translation. In short order, researchers concluded that PER itself figured in the negative feedback and that it operated on transcription (as shown in the right side of Figure 1).

There remained several issues requiring further research, including how exactly PER inhibited its own transcription. No DNA binding region was discovered on PER, so researchers assumed that another molecule must be involved. They searched for it in the same manner as Konopka, producing mutants and screening for affects on circadian rhythms. In this manner, Sehgal et al. (1994) identified a second circadian gene in fruit flies, which they called timeless (tim). In further research they revealed the operation in which it functions: TIM forms a dimer with PER before entering the nucleus and as a dimer inhibits transcription of both per and tim (Vosshall et al., 1994). But since TIM also lacks a DNA binding region, that did not answer the question of how PER (or TIM) regulates its own transcription. An important part of the answer was provided when Vitaterna et al. (1994) employed the same strategy in mice and found a gene they named Clock in which mutations resulted in loss of circadian rhythms. Homologs of Clock were found in fruit flies, and most importantly CLOCK was shown to bind to the promoter of per and tim. PER was now viewed as inhibiting its own transcription by somehow interfering with CLOCK's ability to bind to the per promoter so as to activate its transcription. ${ }^{7}$

${ }^{7}$ The reference to somehow in this sentence makes clear that the discovery of CLOCK was only part of the answer. Only very recently, however, have researchers been able to identify some of the other components that figure in this process and begun to explicate how they work together to alter per transcription (see Duong et al., 2011). 
From these beginnings an extraordinarily successful research program has identified many additional genes and proteins involved in generating circadian rhythms. I will highlight just a few of the discoveries of components of the mammalian clock mechanism that will be relevant to later discussion. In mice, three homologs of per were identified, two of which, Per1 and Per2, were found to function in much the same manner as per in fruit flies. Their proteins were found to dimerize not with TIM but with two variants of cryptochrome (CRY1 and CRY2). BMAL1 was identified as the dimerization partner of CLOCK in mammals, and a number of kinases were discovered that figure in phosphorylating PER and CRY (thereby regulate their degradation and ability to be transported back into the nucleus). Other research revealed the role of Rev-Erbo, a nuclear hormone receptor, as both activated by the CLOCK:BMAL1 dimer and regulating the transcription of Bmal1. In the last decade new systems biology techniques, such as mass screening for proteins that oscillate on a circadian basis; one of these was a second nuclear hormone receptor, ROR $\alpha$, whose expression is also activated by CLOCK:BMAL1 but is expressed earlier than RevErb $\alpha$ and inhibits transcription of Bmal1 (discussed in Bechtel, 2012).

With respect to integration, the project of decomposing the clock mechanism brought a wide variety of researchers with additional expertise, especially in genetics and molecular biology, into chronobiology. They did not, however, simply supplant the traditional behavioral research. Rather, they needed to relate their findings to behavioral research to understand how the components generated the phenomenon. Doing this often required introducing an additional technique, mathematical modeling, and integrating it with the strategies of decomposition. This becomes more apparent when we focus on attempts to recompose the clock mechanism.

\section{Recomposing the Circadian Clock Mechanism}

Mechanistic research requires not just a reductionistic perspective, but a holist one as well since understanding a mechanism requires showing how the parts and operations work together to generate the phenomenon. ${ }^{8}$ Figure 1 already represented a first step in recomposition as it showed how the per gene, $\mathrm{mRNA}$, and protein were connected to each other in a negative feedback circuit. A simple diagrammatic recomposition, however, is not sufficient to understand the dynamical behavior of many mechanisms. Engineers had long recognized that negative feedback loops are capable of generating oscillations, but in many cases such oscillations are damped, not self-sustained as required of a clock mechanism. To investigate whether a feedback mechanism would generate sustained oscillations, researchers had to develop computational models that could simulate the behavior of the proposed mechanism. Even before any parts of the clock mechanism had been discovered, Goodwin (1963) had employed computational modeling to investigate the capacity of feedback loops in gene control systems to produce sustained oscillations, and determined that they would do so only if they included at least one non-linear linear reaction. His

${ }^{8}$ In this regard, mechanistic accounts differs from other contemporary characterizations of reduction such as Bickle's (2003) ruthless reduction, which treats accounts at the lowestlevel as sufficient explanations. 
model was what Craver (2007) calls a how possibly model, but once actual clock components were identified Goldbeter (1995) adapted Goodwin's model to the feedback loop proposed by Hardin et al. (1990). His model employed five differential equations characterizing the formation and degradation of each component in the mechanism (per mRNA, PER in three different states of phosphorylation in the cytoplasm, and PER in the nucleus). Figure 2 (left) shows how the first equation in the model represents the creation and degradation of per mRNA. This equation relates two variables: $M$ represents the concentration of per mRNA and $P_{N}$ the concentration of PER in the nucleus with five parameters: $v_{S}$ represents the maximum rate for the accumulation of $M, K_{I}$ is a threshold constant for inhibition, $n$ is the Hill coefficient indicating the minimum number of cooperating molecules required to achieve inhibition, $v_{m}$ is the maximum rate for the degradation of $M$, and $K_{m}$ is the Michaelis constant for the degradation reaction. The Hill coefficient $n$ embodies the non-linearity required to generate sustained oscillations. By supplying what he took to be biologically plausible values for the parameters, Goldbeter demonstrated that the mechanism proposed by Hardin et al. could generate sustained circadian oscillations by showing that when the successive values of per mRNA and the protein PER were plotted in a phase space defined by those two variables, a limit cycle was created (Figure 2, right). This plot shows not only that when the variables assume values on the cycle (dark oval), subsequent values will follow the trajectory around the cycle, but also that when the variables assume values inside or outside the cycle, they will follow a trajectory (transient) towards the cycle.
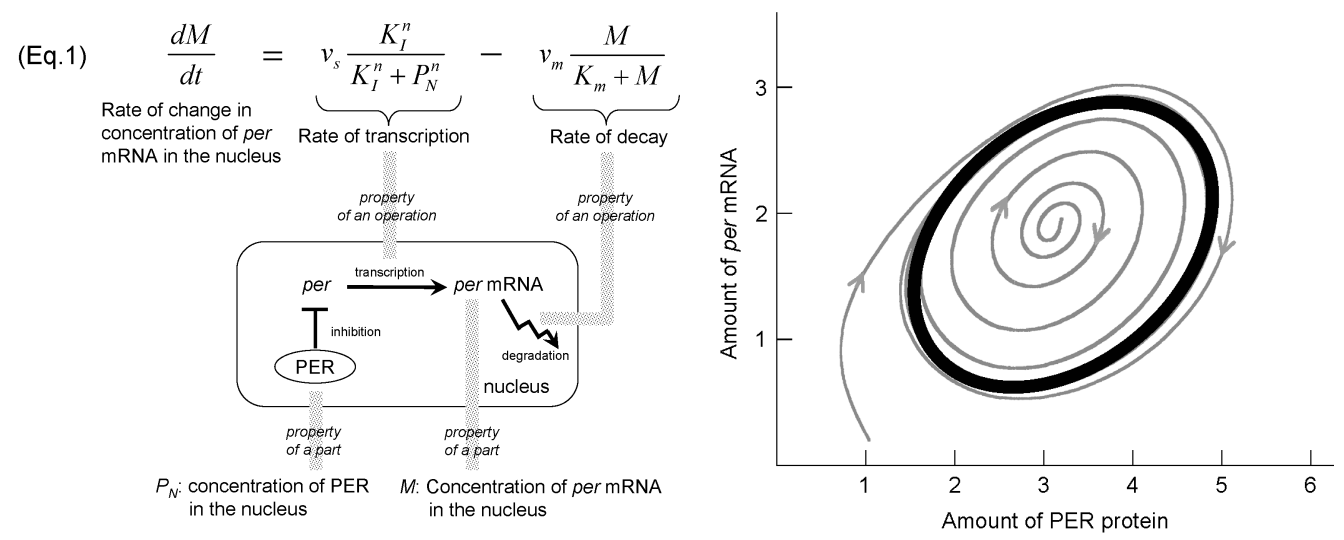

Figure 2. On the left, the first equation in Goldbeter's (1995) model and its relation to the mechanism proposed by Hardin et al (1990). On the right, the limit cycle generated by plotting successive values of per mRNA and PER protein.

These efforts to recompose the clock continued as the other components discussed in the previous section were discovered. Diagrams such as the one on the right in Figure 1 were expanded as in Figure 3 to show where each figured in the mechanism. The fact that RevErb $\alpha$ introduced a second negative feedback loop while ROR $\alpha$ added a positive feedback loop raised the question whether the expanded mechanism would still generate circadian oscillations. To address this, Leloup and Goldbeter added terms and equations to Goldbeter's 1995 model to represent both the fruit fly (Leloup \& Goldbeter, 1998) and the mammalian (Leloup \& Goldbeter, 2004) circadian mechanisms and showed that both generated sustained circadian rhythms. The introduction of two loops raised a further 
question-are both needed to sustain oscillations. This question has been addressed in computational modeling studies in which modelers not only try to replicate the phenomenon to be explained but also examine what would happen in a modified system: in their models they fix concentration values for some components to determine whether others would still oscillate. These efforts have yielded conflicting answers: Smolen et al. (2001) concluded that the feedback loop involving PER was the only one that was sufficient on it own, whereas Relógio et al. (2011) argued that only the one involve Rev-Erbo was sufficient. The differences reflect different assumptions built into the models, but this does not remove the interest in the models since each makes empirical predictions that can guide experimental research (see Bechtel, 2012, for discussion)

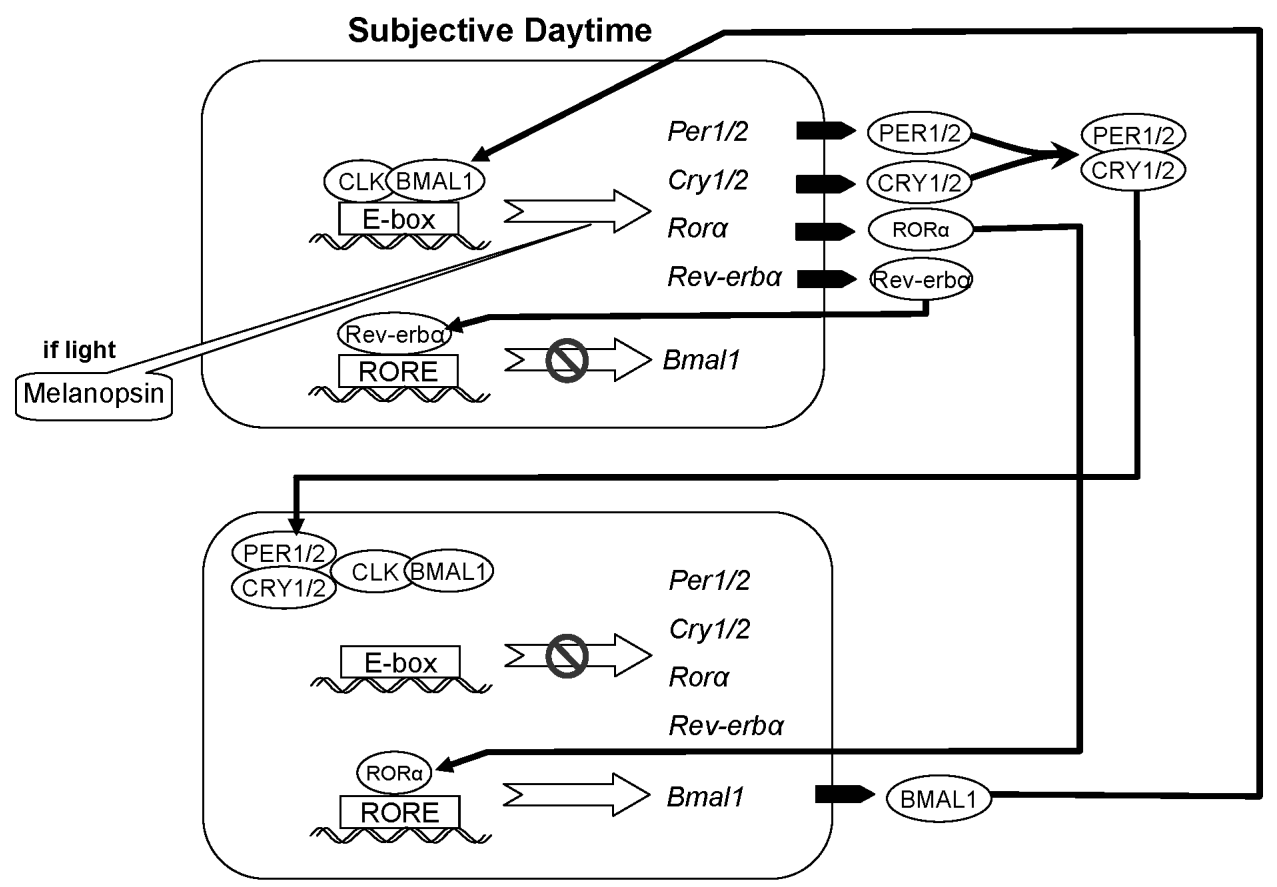

Subjective Nighttime

Figure 3. Representation of the mammalian circadian clock mechanism, incorporating several of the additional components that have been discovered. Of particular note is the fact that Rev-erb $\alpha$ inhibits expression of Bmal1, introducing a second negative feedback loop, while ROR $\alpha$, which is synthesized slightly later, activates the expression of Bmal1, introducing a positive feedback loop.

Efforts to recompose the clock also involved levels above that of the intracellular oscillator. I noted above that the SCN consists of 20,000 neurons on each side of the brain, and a plausible first assumption was that the SCN was simply an aggregate of independent clocks that each functioned much the same. However when Welsh et al. (1995) dissociated SCN neurons on a multi-electrode array (leaving intact some but not all of the connections between neurons), they found that although individual neurons sustained oscillations, their phase and period differed substantially. Some neurons exhibited maximal activity at times when most were quiescent, and the periods of individual oscillators ranged from 21.25 to 26.25 hours with a standard deviation of 1.25 hours. In contrast, when Herzog et al. (2004) 
examined individual cells in slice preparations that preserved more connections, they found little variation. This indicated that the organization within the SCN was crucial to the generation of normal circadian oscillations and pointed to the need to recompose the SCN itself, not just the transcription/translation loops within neurons.

Research decomposing the SCN had already revealed the existence of two separate regions within it characterized partly in terms of the different peptides that are synthesizedprimarily vasoactive intestinal polypeptide (VIP) in a region known as the core and vasopressin in the shell. Aton et al. (2005) identified VIP as the likely agent responsible for generating synchronized behavior. But to determine how the synchronizing signal is disseminated required analyzing the network organization of the SCN. Since direct empirical studies have not revealed the pattern of connectivity in the SCN, researchers have pursed the investigation of the organization of the SCN by developing models advancing different patterns of organization and comparing their performance with that of the SCN itself. The first model, by Gonze et al. (2005), assumed that VPI diffused generally throughout the SCN, effectively assuming that each unit was connected to every other unit. They showed that when the parameter affecting the response to the diffusible compound was set to 0 , the model behaved as the preparation of Welsh et al. had, but when it was set to 0.5 , the oscillators exhibited the synchronization Herzog et al. had found. The assumption of equal diffusion from each node to all others, however, is anatomically unrealistic: instead, individual nodes are more likely to affect those near them. In subsequent research, this group of researchers explored a design intended to capture the relation between core and shell regions of the SCN (Bernard et al., 2007). In this model they were able to replicate the phenomenon that shell neurons tend to oscillate in advance of core neurons, and that when a 12 hour phase shift is introduced, core neurons resynchronize with the day-night cycle within two days but shell neurons require more than ten days. In an separate modeling project, To et al. (2007) showed that by randomly perturbing a parameter specifying basal transcription of Per mRNA they could reproduce Aton et al.'s (2005) finding that in the mouse SCN only 30\% of cells appear capable of sustaining oscillations on their own as well as Ohta et al.'s (2005) finding that in constant light, individual SCN cells continue to oscillate but are desynchronized.

In the last decade researchers have found that a wide variety of real-world networks, including those in the brain, exhibit what Watts and Strogatz (1998) characterized as small-world organization in which most connections are between nearby nodes, but a small number connect distant nodes. Watts and Strogatz argued that such are ideal for information processing since local clusters are able to perform specialized computations but the various local clusters are nonetheless coordinate their activities. Attracted to the idea that the SCN might exhibit small-world properties, Vasalou et al. (2009) developed a computational model in which a parameter determined the percentage of long-range connections (a low value represented small-world organization). On measures of synchronization the small world networks performed comparably to completely connected networks, but with far fewer connections. In their model they were also able to capture three other phenomena observed in experimental studies: compared to networks in which VIP synthesis was shut-off, in the small-world networks (1) the percentage of oscillating 
neurons rises from about $30 \%$ to nearly all, (2) the period is extended from approximately 22 to approximately 24 hours, and (3) the variability in periods is largely eliminated.

In this section I have focused on two steps in the recomposition of the circadian clock mechanism, the recomposition of the transcriptional/translational feedback loop within individual neurons, and the recomposition of the intercellular network within the SCN itself and have emphasized the role computational models have played in such research. In many cases computational models have extended inquiry beyond what can be addressed experimentally, either by simulating manipulations to the mechanism that cannot be performed empirically or by representing modes of organization that have not been demonstrated empirically. Craver (2007) has questioned whether computational models that are not grounded in details of the components of the mechanism are in fact explanatory, claiming that such models only offer how possibly explanations. This criticism, however, fails to recognize the close engagement between computational modeling and empirical investigations on mechanisms. Even when the models advance hypotheses about organization that is beyond what is demonstrated empirically, the goal is to determine how the mechanism is actually organized. This is reflected in such things as the efforts of modelers to evaluate their models by their ability to replicate features of the phenomena, includes features that were not the immediate focus in designing the model and their ability to account for experimental results on perturbed organisms (e.g., those with induced mutations). These extensions beyond the initial phenomena would not be expected unless the model was tracking the actual causal processes occurring in the mechanism. This shows that the modelers' objective is to identify the actual organization that is responsible for the phenomena generated by the circadian mechanism found in living organisms. Moreover, the computational model is the vehicle for representing this organization and determining its effects. The computational model is integral to the explanation (see also Brigandt, this issue, in press).

Efforts to recompose the circadian mechanism have not topped-out at the level of the SCN. Once researchers identified of the molecular components of the clock, they found that the same components were present in other cells of the body and indeed these cells sustained circadian oscillations but, without the SCN, failed to synchronize. The main output of the SCN appears to be to provide a signal that synchronizes clock mechanisms throughout the organism (Davidson et al., 2004). Researchers are also discovering feedback processes whereby activities elsewhere in the body, which are regulated by the SCN, also modulate the circadian oscillations in the SCN. One clear example involves heme, whose synthesis in the liver is under circadian control, but which feeds back to modulate the function of REVERB $\alpha$ in promoting BMAL1 expression (Yin et al., 2007). Similarly, $\mathrm{NAD}^{+}$biosynthesis is under circadian control, but $\mathrm{NAD}^{+}$-levels are controlled by ongoing metabolic processes. $\mathrm{NAD}^{+}$concentration in turn affects the activity of sirtuin 1, which figures in the chromatin remodeling activities through which CLOCK activates Per and Cry transcription (Nakahata et al., 2008). Understanding circadian rhythms in organisms requires integrating knowledge about the intracellular oscillators, the network organization in the SCN, and the interactions with the rest of the organism. By emphasizing the importance of recomposing mechanisms, the mechanistic framework provides a means of integrating research on the various operations that contribute to circadian behavior through a hierarchy of 
mechanisms; accordingly, it is an appropriate framework for philosophers seeking to understand ways in which these scientific investigations are integrated.

\section{Relating the Circadian Clock to Health and Disease}

So far I have focused on how the mechanistic framework serves to integrate circadian research at levels of organization from the molecular to that of the whole-organism. As humans, we are interested not just in the normal functioning of our bodies, but ways in which disease occurs and how it might be prevented or ameliorated. That circadian rhythms are important for healthy function is not surprising-one of the functions of circadian rhythms is to coordinate physiological and behavioral activities with conditions that change with the rotation of the earth. Such coordination requires anticipating these conditions in advance by, for example, synthesizing appropriate genes and there is strong evidence that organisms with circadian clocks with the same period as the light-dark cycle of their environment enjoy greater evolutionary fitness (Woelfle et al., 2004). Research is increasingly revealing specific ways in which circadian rhythms and their perturbations affect human health and disease, and the impetus to understand these linkages is further extending the exploration of connections between the clock mechanism and other physiological mechanisms noted in the examples at the end of the last section.

There has been a long tendency in clinical medicine to neglect circadian rhythms. In particular, many physicians ignore the fact that the physiological measures they employ vary on a circadian cycle. An example is core body temperature, which was already shown to vary by over a degree Celsius in the course of a 24-hour period in Wunderlich's (1868) study that established $37^{\circ} \mathrm{C}$. $\left(98.6^{\circ} \mathrm{F}\right.$.) as normal body temperature. (For historical analysis, see Mackowiak \& Worden, 1994.) Wunderlich had collected multiple recordings per day of body temperature from over 25,000 individuals and found that between 2 and $8 \mathrm{AM}$ mean temperature in his sample was $36.2^{\circ} \mathrm{C}$. (97.2 ${ }^{\circ} \mathrm{F}$.) while between 4 and $9 \mathrm{PM}$ it was $37.5^{\circ} \mathrm{C}$. $\left(99.5^{\circ} \mathrm{F}\right.$.). Of his results, only $37^{\circ} \mathrm{C}$., the mean of over one million reports averaged over all times, has entered into the use of body temperature as an indicator of health. Other physiological measures, such as blood pressure are also under circadian control-blood pressure is lowest in the late night and the increased heart rate following waking may be responsible for the higher rate of strokes and heart attacks during morning hours compared to the rest of the day (Elliott, 1998).

Sleep is the phenomenon most widely associated with circadian rhythms. Although a different mechanism determines the amount an animal sleeps per day, lesions to the SCN disrupt the timing of sleep. There are two well-delineated circadian sleep pathologies: advanced sleep phase syndrome (ASPS) and delayed sleep phase syndrome (DSPS). ASPS patients tend to go to sleep between 5:30 and 8:30 PM and wake up between 1:00 and 5:30 AM. Conversely, individuals with DSPS prefer to go to sleep between 3 and 6 AM and get up between $11 \mathrm{AM}$ and $2 \mathrm{PM}$. These conditions have no effects on either the quality or quantity of sleep when individuals are allowed to set their own sleep time but make it very 
difficult for afflicted individuals to function given the dominate patterns of daily life in our culture (for a review, see Sack et al., 2007). ${ }^{9}$

Clear connections to the circadian clock mechanism have been found in the case of ASPS. Toh et al. (2001) found ASPS in several members of a Utah family and performed a genetic screen that revealed a point mutation (a serine-to-glycine mutation at position 662) in the Per2 gene. Position 662 is where casein kinase I (CKI) acts to phosphorylate PER2, preparing it either for degradation or nuclear transport. The mutation results in hypophosphorylation of PER2. In a second family the mutation was traced to CKI $\delta$ and also results in hypophosphorylation of PER2 (Xu et al., 2005). Subsequent research has supported the hypothesis that reduced phosphorylation yields less nuclear PER either as a result of increased degradation or reduced retention in the nucleus (Takahashi et al., 2008). Not only can alterations in the circadian clock alter sleep activity, but there is also evidence that altered sleep itself can affect the operation of the clock (Deboer et al., 2003; Deboer et al., 2007).

One extremely active field of clinical investigation that is increasingly integrated with research on circadian mechanisms focuses on the effects of disrupters of circadian behavior such as shift work and jet lag. An important feature of the circadian clock is its ability to entrain to the local light-dark cycle (needed to keep a clock whose period is only approximately 24-hour in phase with its environment and to adjust to seasonal differences), but this ability allows for adjustments of only about one hour per day. With a six-hour phase advance (corresponding to travel from New York to Paris), it takes peripheral oscillators that regulate gene expression a week to fully adjust their phase relation to the new light-dark cycle (Yamazaki et al., 2000). A sudden transition between a night and day work schedule requires similar adjustment.

Since the expression of many genes is under circadian control, the effects of such disruptions can be severe. Numerous epidemiological studies have revealed increased cancer rates in individuals regularly forced to entrain to altered activity cycles. For example, increased risks of breast cancer have been found both among shift worker (Davis et al., 2001) and flight attendants who repeatedly change time zones (Rafnsson et al., 2001). Kubo et al. (2006) demonstrated in a prospective study increased risk of prostate cancer in individuals working rotating shifts. There is also epidemiological evidence of increased risk for non-Hodgkin's lymphoma, endometrial, and colon cancers (Logan \& Sarkar, 2012). (See Plutynski, this issue, for discussion of recent integatory research on cancer.)

Such epidemiological studies don't reveal how disruptions of circadian rhythms transform healthy tissue into cancers, but mechanistic research is currently identifying likely causal linkages. The key mechanism in healthy tissue that is altered in cancer is the cell cycle.

${ }^{9}$ Less extreme than these pathological cases but widespread are the preferences for morning (larks) and evening (owls) activity. These have been linked to variable repetitions of the promoter regions on Per3 (Archer et al., 2010), an ortholog of Per1 and Per2 whose precise function in the clock mechanism is not yet known. 
There are two main phases in the cell cycle, the synthesis (S) phase during which DNA is replicated and the mitotic (M) phase during which the cell divides into daughter cells. These are separated by two gap phases, G1 (between the M and S phase) during which gene expression and protein synthesis occur to prepare for DNA replication and G2 (between the $\mathrm{S}$ and $\mathrm{M}$ phases) during which the cell produces proteins and metabolites needed for cell division. Two families of proteins, cyclins and cyclin-dependent kinases, regulate these phases. Checkpoints are located during the $S$ phase and at the transitions between the G1 and $S$ and between the $G$ and $M$ phases of the cycle at which DNA is inspected for damage and DNA damage response (DDR) pathways either repair the DNA or initiate a process of programmed cell death (apoptosis). If these processes fail, tumors develop.

Stages in the cell cycle, both in single-celled organisms such as Gonyaulax and in multicelled organisms such as mice, are restricted to specific times of day by genes whose expression is regulated in a circadian fashion (Miller et al., 2007). Among the genes whose expression is regulated in a circadian fashion are Wee1, which functions at the G2-M checkpoint (Matsuo et al., 2003), and those involved in DDR pathways, such as p21CIP1 (Gréchez-Cassiau et al., 2008). When the circadian clock is disrupted, so is the cell cycleespecially those mechanisms involved in DNA damage detection and repair. There is also evidence that the circadian clock mechanism is regulated by components of the cell cycle. For example, Per2 expression is regulated by C/EBP $\alpha$ that is induced by p53, a gene active in the G1 DNA damage checkpoint. The two mechanisms, far from being independent, modulate each other.

Not only is there growing evidence of linkages between the cell cycle mechanism and the circadian clock mechanism, but there is also evidence of specific linkages by which circadian factors affect cancer. Following up on observations of enlarged, hyperplastic salivary glands in older Per2 mutant mice, Fu et al. (2002) established that Per2 null mutants exposed to $\gamma$-radiation developed spontaneous lymphomas at an increased rate. This pointed to a role in DDR for Per2 by regulating the kinase ataxia telangiectasia mutated (ATM), which functions as a DNA damage signal transducer that initiates DDR (Zhao \& Lee, 2010). Fu et al. also showed that several genes involved in cell-cycle regulation-Cyclin D1, Cyclin A, Mdm02, Gadd45 $\alpha$, and c-Myc-are also deregulated in Per2 mutants. Subsequently Hua et al. (2006) showed that Per2 overexpression in cancer cells inhibited cell proliferation and promotes apoptosis. Other studies have found that Per2 (as well as Per 1 and Per3) down regulation results in a variety of other cancers and that elevated Per2 can protect cells from environmental damage that induces tumorigenesis (see Zhao \& Lee, 2010, for details).

Cancer is not the only health effect of disrupted circadian rhythms. Castanon-Cervantes et al. (2010) showed effects on the immune system: following experimentally induced jet lag, mice fail to produce a coordinated inflammatory response to lipopolysaccharides from bacteria. There is, moreover, clear evidence of connections between circadian mechanisms and those involved in immune system responses involved in defense against bacterial and viral infections. The paraventricular nucleus has been shown to be an important intermediary between the SCN and immune system. It governs autonomic inputs to various 
lymphoid organs such as the thymus, spleen, and lymph nodes. The spleen, for example, mediates activity of natural killer cells, macrophages, and other lymphocytes. There is also evidence that the arcuate nucleus also mediates immune responses through the endogenous opioid beta-endorphin. Finally, melatonin and glucocorticoids oscillate in opposing phase to one another and further regulate immune function. Research on Per2 mutants demonstrates lowered expression of interferon-gamma. This is just one of several factors that are regulated in natural killer cells so as to be at maximal levels during awake periods when an organism is most likely to be exposed to infection and disease. Finally, there is evidence that oscillations in immune factors also regulate the SCN, generating phase advances or delays of circadian rhythms depending on their timing. (See Logan \& Sarkar, 2012, for a review of these findings.)

Adopting a highly segregated view of a particular mechanism is often the only productive way to initiate research (Wimsatt, 2007). Extreme holism, which emphasizes how all of the components of an organism interact, lacks investigatory strategies that can reveal what the components contribute. But in fact biological mechanisms are seldom as segregated as researchers initially assume. The research on the relation between circadian rhythms and sleep, cancer, and immune function on which I have focused in this section makes clear that the different mechanisms are not independent but interact. Moreover, information about the components parts and operations of the mechanisms has provided clear indications of the points at which the different mechanisms affect each other. Thus, as details of a given mechanism are worked out, researchers are increasingly able to make sense of the ways it interacts with others.

\section{Conclusions}

I have employed research on circadian rhythms in chronobiology as a model field for examining some of the ways integration occurs in modern biology. While not becoming a separate discipline, as cell biology did, chronobiology has developed into an enduring interdisciplinary field. As explored by Brigandt (2010), such integration emerged as investigators pursued specific problems-the goal is not integration for its own sake; rather integration is a means to other scientific ends. I have focused specifically on how the shared adoption of the clock mechanism metaphor has provided the basis for integrating investigations delineating the nature of circadian phenomena, research directed at decomposing the clock mechanism into its component genes and proteins, efforts at recomposing the mechanism, especially through computational models, and inquiries into how the clock mechanism interacts with other biological mechanisms in generating disease. Inquiries using different techniques and operating at different levels of organization provide different pieces of information, but since each is viewed as relevant to understanding how the clock mechanism operates, researchers can relate them into an integrated account.

In discussing the role of mechanism in integrating chronobiology research I have drawn attention to two features that have not been emphasized in the recent discussions of mechanistic explanation. The first is that computational modeling is critical to the project of mechanistic explanation for it is what reveals how a given mechanism will function and 
how it will respond to various manipulations. When the phenomenon itself is dynamic, dynamic mechanistic explanations invoking computational models are required. Second, research on the clock mechanism is increasingly being related to research on disease and therapeutic interventions. The disease consequences of altered circadian rhythms have helped reveal how one biological mechanism is intertwined with others, prompting integration of circadian research with that directed at other mechanisms and that directed at ameliorating disease.

The current understanding of the clock mechanism is far from complete-there are still many features of circadian behavior to be characterized, components of the clock to be identified, ways in which components are organized to be discovered and modeled, and linkages to other physiological mechanisms and consequences for heath and disease to be worked out. Thinking in terms of a clock mechanism both helps to make clear where there are lacunae in current accounts and where new information can enhance and force revisions in these current accounts. The processes of integration are not complete, but still developing. The mechanistic perspective will only apply to some instances of integration in science, but where it does, chronobiology can provide a useful model field for understanding what it can contribute.

\section{Acknowledgment}

This paper has benefited from helpful comments and suggestions by Ingo Brigandt and two anonymous referees. I gratefully acknowledge support from the Social Sciences and Humanities Research Council of Canada (Standard Research Grant 410-2008-0400 to Ingo Brigandt).

\section{References}

Archer, S. N., Carpen, J. D., Gibson, M., Lim, G. H., Johnston, J. D., Skene, D. J., \& Schantz, M. v. (2010). Polymorphism in the PER3 promoter associates with diurnal preference and delayed sleep phase disorder. Sleep, 33, 695-701.

Aton, S. J., Colwell, C. S., Harmar, A. J., Waschek, J., \& Herzog, E. D. (2005). Vasoactive intestinal polypeptide mediates circadian rhythmicity and synchrony in mammalian clock neurons. Nature Neuroscience, 8, 476-483.

Bechtel, W. (1986). The nature of scientific integration. In W. Bechtel (Ed.), Integrating scientific disciplines (pp. 3-52). Dordrecht: Martinus Nijhoff.

Bechtel, W. (2006). Discovering cell mechanisms: The creation of modern cell biology. Cambridge: Cambridge University Press.

Bechtel, W. (2012). From molecules to networks: Adoption of systems approaches in circadian rhythm research In H. Andersen, D. Dieks, W. J. Gonzalez, T. Uebel \& G. Wheeler (Eds.), New challenges to philosophy of science: Springer.

Bechtel, W., \& Abrahamsen, A. (2005). Explanation: A mechanist alternative. Studies in History and Philosophy of Biological and Biomedical Sciences, 36, 421-441.

Bechtel, W., \& Abrahamsen, A. (2009). Decomposing, recomposing, and situating circadian mechanisms: Three tasks in developing mechanistic explanations. In H. Leitgeb \& A. 
Hieke (Eds.), Reduction and elimination in philosophy of mind and philosophy of neuroscience (pp. 173-186). Frankfurt: Ontos Verlag.

Bechtel, W., \& Abrahamsen, A. (2010). Dynamic mechanistic explanation: Computational modeling of circadian rhythms as an exemplar for cognitive science. Studies in History and Philosophy of Science Part A, 41, 321-333.

Bechtel, W., \& Abrahamsen, A. (2011). Complex biological mechanisms: Cyclic, oscillatory, and autonomous. In C. A. Hooker (Ed.), Philosophy of complex systems. Handbook of the philosophy of science (Vol. 10, pp. 257-285). New York: Elsevier.

Bechtel, W., \& Hamilton, A. (2007). Reduction, integration, and the unity of science: Natural, behavioral, and social sciences and the humanities. In T. Kuipers (Ed.), Philosophy of science: Focal issues (pp. 377-430). New York: Elsevier.

Bechtel, W., \& Richardson, R. C. (1993/2010). Discovering complexity: Decomposition and localization as strategies in scientific research. Cambridge, MA: MIT Press. 1993 edition published by Princeton University Press.

Bernard, S., Gonze, D., Čajavec, B., Herzel, H., \& Kramer, A. (2007). Synchronization-induced rhythmicity of circadian oscillators in the suprachiasmatic nucleus. PLoS Computational Biology, 3, e68.

Bickle, J. (2003). Philosophy and neuroscience: A ruthlessly reductive account. Dordrecht: Kluwer.

Brigandt, I. (2010). Beyond reduction and pluralism: Toward an epistemology of explanatory integration in biology. Erkenntnis, 73, 295-311.

Brigandt, I. (in press). Evolutionary developmental biology and the limits of philosophical accounts of mechanistic explanation. In P.-A. Braillard \& C. Malatere (Eds.), Explanation in biology: An enquiry into the diversity of explanatory patterns in the life sciences. Berlin: Springer.

Brigandt, I. (this issue). Systems biology and the integration of mechanistic explanation and mathematical explanation. Studies in History and Philosophy of Biological and Biomedical Sciences.

Brigandt, I., \& Love, A. C. (2012). Reduction in biology. In E. N. Zalta (Ed.), Stanford Encyclopedia of Philosophy.

Bünning, E. (1958). Die Physiologische Uhr. Berlin: Springer-Verlag.

Bünning, E. (1960). Opening address: Biological clocks. Cold Spring Harbor Symposia on Quantitative Biology, 25, 1-9.

Castanon-Cervantes, O., Wu, M., Ehlen, J. C., Paul, K., Gamble, K. L., Johnson, R. L., Besing, R. C., Menaker, M., Gewirtz, A. T., \& Davidson, A. J. (2010). Dysregulation of inflammatory responses by chronic circadian disruption. The Journal of Immunology, 185, 5796-5805.

Craver, C. F. (2005). Beyond reduction: Mechanisms, multifield integration and the unity of neuroscience. Studies in History and Philosophy of Biological and Biomedical Sciences, 36, 373-395.

Craver, C. F. (2007). Explaining the brain: Mechanisms and the mosaic unity of neuroscience. New York: Oxford University Press.

Daan, S. (2010). A history of chronobiological concepts. In U. Albrecht (Ed.), The Circadian Clock (Vol. 12, pp. 1-35): Springer New York.

Darden, L. (2006). Reasoning in biological discoveries. Cambridge: Cambridge University Press. 
Davidson, A. J., Yamazaki, S., \& Menaker, M. (2004). SCN: Ringmaster of the circadian circus or conductor of the circadian orchestra? In D. J. Chadwick \& J. A. Goode (Eds.), Molecular clocks and light signalling (pp. 110-125). Chichester, UK: John Wiley.

Davis, S., Mirick, D. K., \& Stevens, R. G. (2001). Night shift work, Light at night, and risk of breast cancer. Journal of the National Cancer Institute, 93, 1557-1562.

De Mairan, J.-J. d. O. (1729). Observation Botanique. Histoire de l'Academie Royale Sciences, 35.

Deboer, T., Détári, L., \& Meijer, J. H. (2007). Long term effects of sleep deprivation on the mammalian circadian pacemaker. Sleep, 30, 257-262.

Deboer, T., Vansteensel, M. J., Detari, L., \& Meijer, J. H. (2003). Sleep states alter activity of suprachiasmatic nucleus neurons. Nature Neuroscience, 6, 1086-1090.

Duong, H. A., Robles, M. S., Knutti, D., \& Weitz, C. J. (2011). A molecular mechanism for circadian clock negative feedback. Science, 332, 1436-1439.

Edmunds, L. N. (1988). Cellular and molecular bases of biological clocks: Models and mechanisms for circadian timekeeping. New York: Springer-Verlag.

Elliott, W. J. (1998). Circadian variation in the timing of stroke onset : A meta-analysis. Stroke, 29, 992-996.

Feldman, J. A., \& Hoyle, M. N. (1973). Isolation of circandian clock mutants of Neurospora crasa. Genetics, 75, 605-613.

Fu, L., Pelicano, H., Liu, J., Huang, P., \& Lee, C. C. (2002). The circadian gene Period2 plays an important role in tumor suppression and DNA damage response in vivo. Cell, 111, 41-50.

Gerson, E. (this issue). Integration of specialties: an institutional and organizational view. Studies in History and Philosophy of Biological and Biomedical Sciences.

Goldbeter, A. (1995). A model for circadian oscillations in the Drosophila Period protein (PER). Proceedings of the Royal Society of London. B: Biological Sciences, 261, 319324.

Gonze, D., Bernard, S., Waltermann, C., Kramer, A., \& Herzel, H. (2005). Spontaneous synchronization of coupled circadian oscillators. Biophysical Journal, 89, 120-129.

Goodwin, B. C. (1963). Temporal organization in cells; a dynamic theory of cellular control processes. London: Academic.

Gréchez-Cassiau, A., Rayet, B., Guillaumond, F., Teboul, M., \& Delaunay, F. (2008). The Circadian Clock Component BMAL1 Is a Critical Regulator of p21WAF1/CIP1 Expression and Hepatocyte Proliferation. Journal of Biological Chemistry, 283, 45354542.

Halberg, F., Halberg, E., Barnum, C. P., \& Bittner, J. J. (1959). Physiologic 24-Hour Periodicity in Human Beings and Mice, the Lighting Regimen, and Daily Routine. In R. B. Withrow (Ed.), Photoperiodism and Related Phenomena in Plants and Animals (pp. 803-878). Washington, DC: American Association for the Advancement of Science.

Hardin, P. E., Hall, J. C., \& Rosbash, M. (1990). Feedback of the Drosophila period gene product on circadian cycling of its messenger RNA levels. Nature, 343, 536-540.

Herzog, E. D., Aton, S. J., Numano, R., Sakaki, Y., \& Tei, H. (2004). Temporal precision in the mammalian circadian system: A reliable clock from less reliable neurons. Journal of Biological Rhythms, 19, 35-46. 
Hua, H., Wang, Y., Wan, C., Liu, Y., Zhu, B., Yang, C., Wang, X., Wang, Z., CornelissenGuillaume, G., \& Halberg, F. (2006). Circadian gene mPer2 overexpression induces cancer cell apoptosis. Cancer Science, 97, 589-596.

Inouye, S.-I. T., \& Kawamura, H. (1979). Persistence of circadian rhythmicity in a mammalian hypothalamic "island" containing the suprachiasmatic nucleus. Proceedings of the National Academy of Sciences (USA), 76, 5962-5966.

Johnson, M. S. (1939). Effect of continuous light on periodic spontaneous activity of whitefooted mice (Peromyscus). Journal of Experimental Zoology, 82, 315-328.

Konopka, R. J., \& Benzer, S. (1971). Clock mutants of Drosophila melanogaster. Proceedings of the National Academy of Sciences (USA), 89, 2112-2116.

Kubo, T., Ozasa, K., Mikami, K., Wakai, K., Fujino, Y., Watanabe, Y., Miki, T., Nakao, M., Hayashi, K., Suzuki, K., Mori, M., Washio, M., Sakauchi, F., Ito, Y., Yoshimura, T., \& Tamakoshi, A. (2006). Prospective cohort study of the risk of prostate cancer among rotating-shift workers: Findings from the Japan collaborative cohort study. American Journal of Epidemiology, 164, 549-555.

Leloup, J.-C., \& Goldbeter, A. (1998). A model for circadian rhythms in Drosophila incorporating the formation of a complex between the PER and TIM proteins. Journal of Biological Rhythms, 13, 70-87.

Leloup, J.-C., \& Goldbeter, A. (2004). Modeling the mammalian circadian clock: Sensitivity analysis and multiplicity of oscillatory mechanisms. Journal of Theoretical Biology, 230, 541-562.

Logan, R. W., \& Sarkar, D. K. (2012). Circadian nature of immune function. Molecular and Cellular Endocrinology, 249, 82-90.

Machamer, P., Darden, L., \& Craver, C. F. (2000). Thinking about mechanisms. Philosophy of Science, 67, 1-25.

Mackowiak, P. A., \& Worden, G. (1994). Carl Reinhold August Wunderlich and the evolution of clinical thermometry. Clinical Infectious Diseases, 18, 458-467.

Matsuo, T., Yamaguchi, S., Mitsui, S., Emi, A., Shimoda, F., \& Okamura, H. (2003). Control mechanism of the circadian clock for timing of cell division in vivo. Science, 302, 255259.

Miller, B. H., McDearmon, E. L., Panda, S., Hayes, K. R., Zhang, J., Andrews, J. L., Antoch, M. P., Walker, J. R., Esser, K. A., Hogenesch, J. B., \& Takahashi, J. S. (2007). Circadian and CLOCK-controlled regulation of the mouse transcriptome and cell proliferation. Proceedings of the National Academy of Sciences, 104, 3342-3347.

Moore, R. Y., \& Eichler, V. B. (1972). Loss of a circadian adrenal corticosterone rhythm following suprachiasmatic lesions in the rat. Brain Research, 42, 201-206.

Moore, R. Y., \& Lenn, N. J. (1972). A retinohypothalamic projection in the rat. The Journal of Comparative Neurology, 146, 1-14.

Nakahata, Y., Kaluzova, M., Grimaldi, B., Sahar, S., Hirayama, J., Chen, D., Guarente, L. P., \& Sassone-Corsi, P. (2008). The NAD+-dependent deacetylase SIRT1 modulates CLOCK-mediated chromatin remodeling and circadian control. Cell, 134, 329-340.

Ohta, H., Yamazaki, S., \& McMahon, D. G. (2005). Constant light desynchronizes mammalian clock neurons. Nature Neuroscience, 8, 267-269.

Plutynski, A. (this issue). Integrative perspectives on cancer. Studies in History and Philosophy of Biological and Biomedical Sciences. 
Rafnsson, V., Tulinius, H., Jónasson, J., \& Hrafnkelsson, J. (2001). Risk of breast cancer in female flight attendants: a population-based study (Iceland). Cancer Causes and Control, 12, 95-101.

Ralph, M. R., Foster, R. G., Davis, F. C., \& Menaker, M. (1990). Transplanted suprachiasmatic nucleus determines circadian period. Science, 247, 975-978.

Ralph, M. R., \& Menaker, M. (1988). A mutation of the circadian system in golden hamsters. Science, 241, 1225-1227.

Relógio, A., Westermark, P. O., Wallach, T., Schellenberg, K., Kramer, A., \& Herzel, H. (2011). Tuning the mammalian circadian clock: Robust synergy of two loops. PLoS Computational Biology, 7, e1002309.

Richter, C. P. (1967). Sleep and activity: Their relation to the 24 hour clock. In S. S. Kety, E. V. Evarts \& H. L. Williams (Eds.), Sleep and altered states of consciousness (pp. 8-29). Baltimore: Williams and Wilkins.

Sack, R. L., Auckley, D., Auger, R. R., Carskadon, M. A., Wright Jr., K. P., Vitiello, M. V., \& Zhdanova, I. V. (2007). Circadian rhythm sleep disorders: Part Ii, Advanced sleep phase disorder, Delayed sleep phase disorder, free-running disorder, and irregular sleep-wake rhythm. Sleep, 30, 1484-1501.

Sehgal, A., Price, J. L., Man, B., \& Young, M. W. (1994). Loss of circadian behavioral rhythms and per RNA oscillations in the Drosophila mutant timeless. Science, 263, 1603-1606.

Simon, H. A. (1962). The architecture of complexity: hierarchic systems. Proceedings of the American Philosophical Society, 106, 467-482.

Smolen, P., Baxter, D. A., \& Byrne, J. H. (2001). Modeling circadian oscillations with interlocking positive and negative feedback loops. Journal of Neuroscience, 21, 66446656.

Stephan, F. K., \& Zucker, I. (1972). Circadian rhythms in drinking behavior and locomotor activity of rats Are eliminated by hypothalamic lesions. Proceedings of the National Academy of Sciences (USA), 69, 1583-1586.

Takahashi, J. S., Hong, H.-K., Ko, C. H., \& McDearmon, E. L. (2008). The genetics of mammalian circadian order and disorder: implications for physiology and disease. Nat Rev Genet, 9, 764-775.

To, T.-L., Henson, M. A., Herzog, E. D., \& Doyle, F. J., III. (2007). A molecular model for intercellular synchronization in the mammalian circadian clock. Biophysical Journal, 92, 3792-3803.

Toh, K. L., Jones, C. R., He, Y., Eide, E. J., Hinz, W. A., Virshup, D. M., Ptáček, L., J., \& Fu, Y.-H. (2001). An $h$ Per2 phosphorylation site mutation in familial advanced sleep phase syndrome. Science, 291, 1040-1043.

Vasalou, C., Herzog, E. D., \& Henson, M. A. (2009). Small-world network models of intercellular coupling predict enhanced synchronization in the suprachiasmatic nucleus. Journal of Biological Rhythms, 24, 243-254.

Virey, J.-J. (1814). Ephémerides de la vie humaine, ou recherches sur la révolution journaliere et la periodicité de ses phénomènes dans la santé et les malades. Paris: Sorbonne.

Vitaterna, M. H., King, D. P., Chang, A.-M., Kornhauser, J. M., Lowrey, P. L., McDonald, J. D., Dove, W. F., Pinto, L. H., Turek, F. W., \& Takahashi, J. S. (1994). Mutagenesis and mapping of a mouse gene, Clock, essential for circadian behavior. Science, 264, 719725. 
Vosshall, L. B., Price, J. L., Sehgal, A., Saez, L., \& Young, M. W. (1994). Block in nuclear localization of period protein by a second clock mutation, timeless. Science, 263, 1606-1609.

Watts, D., \& Strogratz, S. (1998). Collective dynamics of small worlds. Nature, 393, 440-442.

Welsh, D. K., Logothetis, D. E., Meister, M., \& Reppert, S. M. (1995). Individual neurons dissociated from rat suprachiasmatic nucleus express independently phased circadian firing rhythms. Neuron, 14, 697-706.

Wimsatt, W. C. (1974). Complexity and organization. In K. F. Schaffner \& R. S. Cohen (Eds.), PSA 1972 (pp. 67-86). Dordrecht: Reidel.

Wimsatt, W. C. (2007). Re-engineering philosophy for limited beings: Piecewise approximations to reality. Cambridge, MA: Harvard University Press.

Woelfle, M. A., Ouyang, Y., Phanvijhitsiri, K., \& Johnson, C. H. (2004). The adaptive value of circadian clocks: An experimental assessment in cyanobacteria. Current Biology, 14, 1481-1486.

Wunderlich, K. R. A. (1868). Das Verhalten der Eigenwärme in Krankheiten. Leipzig: Otto Wigard.

Xu, Y., Padiath, Q. S., Shapiro, R. E., Jones, C. R., Wu, S. C., Saigoh, N., Saigoh, K., Ptacek, L. J., \& $\mathrm{Fu}, \mathrm{Y}$.-H. (2005). Functional consequences of a CKI $\delta$ mutation causing familial advanced sleep phase syndrome. Nature, 434, 640-644.

Yamazaki, S., Numano, R., Abe, M., Hida, A., Takahashi, R.-i., Ueda, M., Block, G. D., Sakaki, Y., Menaker, M., \& Tei, H. (2000). Resetting central and peripheral circadian oscillators in transgenic rats. Science, 288, 682-685.

Yin, L., Wu, N., Curtin, J. C., Qatanani, M., Szwergold, N. R., Reid, R. A., Waitt, G. M., Parks, D. J., Pearce, K. H., Wisely, G. B., \& Lazar, M. A. (2007). Rev-erb $\alpha$, a heme sensor that coordinates metabolic and circadian pathways. Science, 318, 1786-1789.

Zhao, Z., \& Lee, C. C. (2010). Circadian clock, Cell cycle and cancer. In U. Albrecht (Ed.), The Circadian Clock (Vol. 12, pp. 139-155): Springer New York. 